\title{
Cardiotocografia computadorizada em gestaçōes complicadas pelo diabete melito pré-gestacional: padrões da frequêencia cardiaca em fetos grandes para a idade gestacional
}

\begin{abstract}
Computerized cardiotocography in pregnancies complicated by pregestational diabetes mellitus: heart rate patterns in large for gestational age fetuses
\end{abstract}

\author{
Roseli Mieko Yamamoto Nomura ${ }^{1}$, Verbênia Nunes Costa ${ }^{2}$, Kathia Sakamoto \\ Carlos Alberto Maganha ${ }^{3}$, Seizo Miyadahira ${ }^{1}$, Marcelo Zugaib ${ }^{4}$
}

\section{RESUMO}

Objetivo: verificar os padrões da freqüência cardíaca de fetos grandes para a idade gestacional (GIG), em gestantes com diabete melito pré-gestacional. Métodos: sessenta e quatro gestantes diabéticas pré-gestacionais foram avaliadas semanalmente quanto à vitalidade fetal. Os critérios de inclusão foram: diagnóstico pré-gestacional de diabetes melito, gestação única, feto vivo, ausência de anomalia fetal e cardiotocografia computadorizada realizada na $37^{a}$ semana. Os critérios de exclusão foram: diagnóstico pós-natal de anomalia fetal e parto não realizado na instituição. Os padrões da freqüência cardíaca fetal (FCF) foram investigados pela cardiotocografia computadorizada (Sistema-8002 Sonicaid). Os parâmetros foram analisados de acordo com a classificação pela adequação do peso do recém-nascido em GIG (acima do percentil 90 para a idade gestacional). Os parâmetros cardiotocográficos incluíram: FCF basal, acelerações, episódios de alta variação, episódios de baixa variação e variação de curto prazo. Resultados: do total, 42 pacientes preencheram os critérios propostos. Houve 10 recém-nascidos GIG $(23,8 \%)$. A cardiotocografia apresentou resultado normal em todos os casos. As acelerações da FCF (superiores a $15 \mathrm{bpm}$ ) estavam presentes em 7 (70\%) dos casos GIG e em 29 (90,6\%) dos casos não GIG $(\mathrm{p}=0,135)$. A freqüência dessas acelerações foi maior no grupo não $\operatorname{GIG}(1,5 \pm 1,3$ acelerações/10 min) quando comparado ao grupo GIG $(0,8 \pm 0,9$ acelerações $/ 10 \mathrm{~min}, \mathrm{p}=0,04$, teste de Mann-Whitney). Os episódios de alta variação foram detectados em todos os casos. A média da variação nesses episódios foi diferente no grupo GIG $(16,2 \pm 2,5 \mathrm{bpm})$ quando comparado ao não GIG (19,7 4 4,2 bpm, p=0,02, teste de Mann-Whitney). Conclusões: os padrões da FCF verificados em fetos não GIG (maior freqüência de acelerações e a maior variação da FCF em episódios de alta variação) refletem parâmetros comumente analisados pela cardiotocografia tradicional na higidez fetal. Esse fato sugere a existência de padrões indicativos de melhor condição de oxigenação dos fetos menos comprometidos pelos efeitos do diabetes na gravidez.

PALAVRAS-CHAVE: Cardiotocografia; Freqüência cardíaca fetal; Macrossomia fetal; Diabetes mellitus

\section{ABSTRACT}

Purpose: to verify the fetal heart rate (FHR) patterns of large for gestational age (LGA) fetuses in pregnancies at term complicated by pregestational diabetes. Methods: fetal surveillance was performed weekly in 64 fetuses of mothers with pregestational diabetes. Inclusion criteria were: diagnosis of pregestational diabetes mellitus, single pregnancy, alive fetus, absence of fetal anomalies, and computerized cardiotocography performed at the 37th week of gestation. Exclusion criteria included: postnatal diagnosis of fetal anomalies and delivery not performed at the local hospital. The FHR patterns were studied with computerized cardiotocography and the parameters were analyzed according to a fetal weight as LGA (birth weight above percentile 90). The cardiotocography parameters included: basal FHR, episodes of high variation, episodes of

Trabalho realizado na Clínica Obstétrica do Hospital das Clínicas da Faculdade de Medicina da Universidade de São Paulo - USP - São Paulo (SP) - Brasil.

1 Professor Colaborador Médico do Departamento de Obstetrícia e Ginecologia da Faculdade de Medicina da Universidade de São Paulo - USP - São Paulo (SP) - Brasil.

2 Pós-graduando em Obstetrícia do Departamento de Obstetrícia e Ginecologia da Faculdade de Medicina da Universidade de São Paulo - USP - São Paulo (SP) - Brasil.

3 Médico Assistente da Clínica Obstétrica do Hospital das Clínicas da Faculdade de Medicina da Universidade de São Paulo - USP - São Paulo (SP) - Brasil. 4 Professor Titular do Departamento de Obstetrícia e Ginecologia da Faculdade de Medicina da Universidade de São Paulo - USP - São Paulo (SP) - Brasil. Correspondência: Roseli Mieko Yamamoto Nomura

Rua General Canavarro, 280 - Bairro Campestre - 09070-440 - Santo André - SP - Telefone: (11) 4991-2481 - 4221-8778 - Fax: 4221-8752 - e-mail: roseli.nomura@terra.com.br

Recebido em: 20/10/2005

Aceito com modificações em: 28/11/2005

Rev Bras Ginecol Obstet. 2005;27(12): 712-8 
low variation, and short-term variation. Results: forty-two patients fulfilled the proposed criteria. Ten (23.8\%) newborns were LGA. Normal criteria were met in all performed examinations. FHR accelerations (above $15 \mathrm{bpm})$ were present in $7(70 \%)$ LGA cases and in $29(90.6 \%)$ non-LGA $(\mathrm{p}=0.135)$. Accelerations were more frequent in the non-LGA group $(1.5 \pm 1.3$ accelerations $/ 10 \mathrm{~min})$ when compared to LGA group $(0.8 \pm 0.9$ accelerations $/ 10 \mathrm{~min}, \mathrm{p}=0.04$, Mann-Whitney test). The high variation episodes were detected in all cases. The mean FHR variation in these episodes was different in the LGA group $(16.2 \pm 2.5 \mathrm{bpm})$ when compared to the non-LGA group ( $19.7 \pm 4.2 \mathrm{bpm}, \mathrm{p}=0.02$, Mann-Whitney test). Conclusion: the FHR patterns of non-LGA (higher frequency of accelerations and higher FHR variation in the high variation episodes) reflect parameters commonly analyzed by traditional cardiotocography of a healthy fetus. This fact appears to confirm the patterns of better oxygen supply to the fetuses less compromised by diabetes in pregnancy.

KEYWORDS: Cardiotocography; Heart rate, fetal; Fetal macrosomia; Diabetes mellitus

\section{Introdução}

A avaliação do bem-estar fetal nas gestações complicadas pelo diabete melito requer cuidados por parte de todos os profissionais que as acompanham ${ }^{1}$. Assegurar o não comprometimento da vitalidade fetal é fundamental para que se atinja o termo com segurança e tranqüilidade. Morbidade e mortalidade perinatais mais elevadas eram observadas em maior proporção nos casos sem acompanhamento clínico adequado ${ }^{2}$, principalmente entre as pacientes com diabete diagnosticado previamente à gravidez, também denominado diabete pré-gestacional ${ }^{3,4}$.

Entre os métodos de avaliação da vitalidade fetal, a cardiotocografia tem sido um dos exames mais empregados nas últimas décadas para o auxílio no seguimento das gestações de alto risco ${ }^{1}$. Por ser exame essencialmente não invasivo, a cardiotocografia tem sido utilizada habitualmente como método de vigilância do bem-estar fetal, principalmente nas gestações complicadas pelo diabete $^{1,4}$. A cardiotocografia computadorizada introduziu uma nova era na análise da freqüência cardiaca fetal $(\mathrm{FCF})^{5,6}$. Esse sistema, dotado de programa para a aquisição direta do sinal dos batimentos cardíacos fetais, possibilita a análise objetiva dos parâmetros da FCF.

Torna-se interessante a possibilidade de estudar, pela cardiotocografia computadorizada, as repercussões do diabete materno sobre a regulação da FCF, principalmente nos casos que venham a apresentar ganho ponderal excessivo e macrossomia no termo. Estes terão maior risco de apresentar complicações no parto e também morbidade neonatal elevada ${ }^{7}$. A análise computadorizada permite avaliar, do ponto de vista funcional, e de modo objetivo, as alterações dos padrões da FCF em gestações comprometidas pelo diabete melito.

Os fetos que apresentam alterações no seu metabolismo em face do descontrole materno são os de maior risco para complicações, tanto no período antenatal, como no pós-natal e ao longo de toda sua existência ${ }^{3,4,7}$. Poucos estudos analisam os padrões da cardiotocografia computadorizada em gestações com diabete ${ }^{8-11}$, e nenhum relaciona os seus resultados às repercussões do diabete sobre o concepto.

Este estudo foi delineado para estabelecer novos conhecimentos a respeito deste método propedêutico na avaliação fetal em gestações que cursam com diabete melito pré-gestacional. Assim, o objetivo deste trabalho consiste em verificar os padrões da freqüência cardíaca nos fetos grandes para a idade gestacional (GIG), condição essa que reflete maior comprometimento fetal como conseqüência do diabete melito na gestação.

\section{Métodos}

Durante o período compreendido entre janeiro de 2000 e junho de 2002 , foram acompanhadas prospectivamente as gestantes com diagnóstico de diabete melito pré-gestacional, no Setor de Vitalidade Fetal da Clínica Obstétrica do Hospital das Clínicas da Faculdade de Medicina da Universidade de São Paulo (HCFMUSP). Este estudo foi aprovado pela Comissão de Ética para Análise de Projetos de Pesquisa (CAPPesq) do HCFMUSP, sob número 425/00.

As pacientes foram selecionadas utilizandose os seguintes critérios de inclusão: diagnóstico pré-gestacional de diabete melito, gestação única, feto vivo, ausência de anomalia fetal e cardiotocografia computadorizada realizada na $37^{\mathrm{a}}$ semana. Os critérios de exclusão foram: diagnóstico pós-natal de anomalia fetal e parto não realizado na instituição. Todas as gestantes foram acompanhadas pelo Setor de Endocrinopatias e Gestação da Clínica Obstétrica do HCFMUSP e consentiram em participar do estudo.

No período analisado, 64 gestantes com diagnóstico de diabetes melito pré-gestacional foram acompanhadas pelo Setor de Vitalidade Fetal. A partir da seleção dos casos, as pacientes foram en- 
caminhadas ao Setor de Vitalidade para iniciarem a propedêutica fetal semanal. Dois casos apresentaram diagnóstico de malformação fetal e não foram incluídos na pesquisa. Foram excluídos 19 casos não submetidos a cardiotocografia na época estabelecida para o estudo e um caso cujo parto não foi realizado nesta instituição, restando para a análise proposta nos objetivos 42 casos. A idade gestacional em que se iniciou o acompanhamento da gestante apresentou média de 29,6 semanas e desvio padrão de 2,4 semanas.

Dezenove pacientes apresentavam diabete tipo $1(45,2 \%)$ e $23(54,8 \%)$ tinham diabete tipo 2 . Os diagnósticos clínicos mais freqüentemente associados na população analisada foram as sindromes hipertensivas, constatadas em seis pacientes $(14,3 \%)$, e o hipotiroidismo, verificado em dois casos $(4,8 \%)$. A idade materna variou de 16 a 45 anos, com média de 31,2 anos, desvio padrão de 7,9 anos e mediana de 32 anos. Quanto à cor da pele, 23 pacientes eram da cor branca $(54,8 \%), 10$ pardas $(23,8 \%)$ e nove pretas $(21,4 \%)$. Em relação aos antecedentes obstétricos, 14 eram nuliparas, correspondendo a $33,3 \%$ dos casos.

O acompanhamento pré-natal foi realizado pelo Setor de Endocrinopatias e Gestação. As pacientes foram categorizadas de acordo com a classificação de Priscilla White, sendo que 20 gestantes $(47,6 \%)$ apresentavam-se na categoria $\mathrm{B}, 10$ na $\mathrm{C} 1(23,8 \%), 10$ na $\mathrm{D}(23,8 \%)$, uma na $\mathrm{F}(2,4 \%) \mathrm{e}$ uma na $\mathrm{R}(2,4 \%)$. O controle glicêmico baseou-se na mensuração dos valores de glicemia capilar total de jejum (normal quando entre 70 e $90 \mathrm{mg}$ / $\mathrm{dL}$ ) e pós-prandial de duas horas (normal quando entre 70 e $120 \mathrm{mg} / \mathrm{dL}$ ). As medidas adotadas para obtenção do controle glicêmico incluíram a dieta fracionada de 35 a $45 \mathrm{cal} / \mathrm{kg}$ de peso ideal por dia e adequação da terapêutica com insulina. Iniciouse com dose única matinal de insulina NPH, que foi ajustada progressivamente no decurso da gestação, acrescentando-se ou não doses noturnas e/ ou insulina de ação rápida pré-prandial. Gestantes de dificil controle foram hospitalizadas até acerto da dose de insulina.

Os dados clínicos da gestação, do parto e os resultados perinatais foram coletados a partir da consulta aos prontuários das pacientes. A idade gestacional no nascimento foi calculada a partir da data da última menstruação, quando esta foi compativel com a idade gestacional estimada pela primeira ultra-sonografia, realizada,no máximo até a vigésima semana de gestação (discordância inferior a 14 dias). Nos casos em que não houve esta concordância, utilizamos a idade gestacional calculada pela primeira ultra-sonografia. Foram investigados os dados relativos ao parto e aos recém-nascidos para melhor caracterização dos grupos. O peso dos recém-nascidos foi aferido logo após o nascimento, assim que o recém-nascido recebia os primeiros cuidados. A adequação do peso do recém-nascido foi avaliada pela comparação do peso no nascimento com os valores normais para a idade gestacional. Foram classificados como GIG os recém-nascidos com peso acima do percentil 90, de acordo com a curva de normalidade desenvolvida por Ramos ${ }^{12}$. Os índices de Apgar do $1^{\circ}$ e $5^{\circ}$ minutos de vida foram considerados alterados quando com valores inferiores a sete.

A cardiotocografia computadorizada foi realizada no período anteparto, com a paciente em posição de semi-Fowler, utilizando-se do aparelho de marca Sonicaid (Sistema 8002, Oxford, Reino Unido).

Nos primeiros 10 minutos de realização do exame, o sistema computadorizado não realiza nenhuma interpretação. Após este período, o programa realiza a primeira análise do traçado, que é revista a cada dois minutos e apresentado na tela do vídeo, até que se atinja o critério de normalidade adotado. A cada análise, o sistema emite um sinal sonoro. O período máximo para realização do traçado é de 60 minutos.

A análise computadorizada baseia-se no estudo da duração dos intervalos de tempo em milissegundos (ms) entre sucessivos batimentos cardiacos fetais. O sistema 8002 analisa o traçado cardiotocográfico em periodos de 3,75 segundos (1/16 de minuto), calculando a FCF média em cada período e também as diferenças entre períodos adjacentes. Os movimentos fetais são registrados pela gestante durante o exame ao acionar dispositivo próprio. As contrações uterinas são detectadas quando ocorre aumento relativo da pressão uterina, superior a $16 \%$ em relação ao tônus basal de repouso, por pelo menos 30 segundos.

Os parâmetros da FCF analisados são: FCF basal, calculada pela média dos intervalos de pulso verificados em todos os periodos de baixa variação (quando não são observados períodos de baixa variação, o cálculo deriva-se de análise estatística); acelerações, identificadas pelo aumento de 10 ou 15 bpm em relação à linha de base com duração superior a 15 segundos; desacelerações, detectadas pela redução maior ou igual a 20 lost beats $^{1}$ em traçados com duração inferior a $30 \mathrm{mi}-$ nutos ou pela redução maior ou igual a 50 lost beats em traçados com duração superior a 30 minutos; variação de curto prazo, que corresponde à média das diferenças dos valores da FCF média (expressa por intervalo de pulso em ms), observada entre os períodos adjacentes de 3,75 segundos; episódios de alta variação, caracterizados quando pelo menos cinco de seis minutos consecutivos do traçado apresentarem variação pico a pico por minuto, superior a $32 \mathrm{~ms}$; variação da FCF nos episódios de alta variação, corresponde à variabilidade 
em batimentos por minuto (bpm) observada nesses episódios, e episódios de baixa variação, definidos quando pelo menos cinco de seis minutos consecutivos do traçado apresentarem variação pico a pico por minuto inferior a $30 \mathrm{~ms}$. A mensuração da desaceleração em unidades denominadas lost beats tem como base o número de batimentos 'perdidos' com a redução da FCF. Uma desaceleração de 20 lost beats corresponde àquela que apresenta queda da FCF de $20 \mathrm{bpm}$ por 60 segundos ou de $40 \mathrm{bpm}$ por 30 segundos $^{5}$.

O exame é interpretado como normal pelo sistema computadorizado quando o critério de Dawes e Redman é atingido. Para que isso ocorra é necessário que as seguintes condições estejam presentes: FCF basal entre 116 e $160 \mathrm{bpm}$; um movimento fetal ou três acelerações; sem evidências de ritmo sinusoidal; a variação de curto prazo superior ou igual a $3 \mathrm{~ms}$; apresentar no mínimo um episódio de alta variação (com variação acima do percentil 1 para a idade gestacional), e uma aceleração ou a variabilidade nos episódios de alta variação superior ao percentil 10, com estimativa da contagem de movimentos fetais por hora superior a 20.

Nesta pesquisa foram analisados os seguintes parâmetros da cardiotocografia computadorizada: critério de normalidade de Dawes e Redman; freqüência de movimentos fetais, avaliados em número de movimentos por hora; FCF basal em bpm; presença de acelerações da FCF de amplitude superiores a $10 \mathrm{bpm}$ e superiores a $15 \mathrm{bpm}$; número de acelerações ( $>10 \mathrm{bpm}$ e $>15 \mathrm{bpm}$ ) a cada 10 minutos; duração dos episódios de alta variação e de baixa variação, em percentual do traçado (porcentagem da duração dos episódios em relação ao tempo total do exame); variação da FCF nos episódios de alta variação, em bpm, e variação de curto prazo em ms.

A análise descritiva das variáveis quantitativas foi realizada pelo cálculo de médias, medianas e desvios-padrão. Para as variáveis qualitativas foram calculadas as freqüências absolutas e relativas. Para a comparação entre os grupos, foram utilizados testes não paramétricos na análise das variáveis contínuas: teste de MannWhitney U para amostras independentes. Para as comparações entre as proporções foram utilizadas análises pelo teste exato de Fisher. Adotou-se como nível de significância o valor $0,05(\alpha=5 \%)$. Com isso, niveis descritivos (p) inferiores a esse valor foram considerados significantes $(\mathrm{p}<0,05)$.

\section{Resultados}

No período analisado, do total de 64 gestantes com diagnóstico de diabete melito pré-gesta- cional que foram acompanhadas pelo Setor de Vitalidade Fetal, 42 pacientes $(65,6 \%)$ realizaram a cardiotocografia computadorizada na $37^{\mathrm{a}}$ semana de gestação. Na Tabela 1 estão expostas as características maternas e os resultados perinatais de acordo com a adequação do peso do recém-nascido. Houve 10 recém-nascidos GIG $(23,8 \%)$ e 32 não GIG $(76,2 \%)$. Não foram observadas diferenças significativas quanto à idade e paridade maternas, entre os grupos que apresentaram ou não recémnascidos GIG. Da mesma forma, os dados perinatais não mostram diferenças quanto à idade gestacional no nascimento, ao tipo de parto ou aos indices de Apgar do $1^{\circ}$ minuto. Todos os recémnascidos apresentaram índices de Apgar do $5^{\circ}$ minuto superior a sete.

Tabela 1 - Médias e desvios-padrão da idade materna, idade gestacional no nascimento, peso dos recém-nascidos (RN) e distribuição segundo a paridade, o tipo de parto, sexo dos $\mathrm{RN}$ e índice de Apgar do primeiro minuto em casos de diabete melito pré-gestacional de acordo com a adequação do peso do recém-nascido.

\begin{tabular}{|c|c|c|c|c|c|}
\hline \multirow[b]{3}{*}{$\begin{array}{l}\text { Idade materna, anos, } \\
\text { média (DP) }\end{array}$} & \multicolumn{5}{|c|}{ Adequação do peso do RN } \\
\hline & \multicolumn{2}{|c|}{ GIG (n = 10) } & \multicolumn{2}{|c|}{ Não GIG (n = 32) } & \multirow{2}{*}{$\frac{p}{0,409^{*}}$} \\
\hline & 33,0 & $(8,0)$ & 30,6 & $(8,0)$ & \\
\hline Nulíparas, n (\%) & \multicolumn{2}{|c|}{$2(20,0 \%)$} & \multicolumn{2}{|c|}{$12(37,5 \%)$} & $0,451^{* *}$ \\
\hline $\begin{array}{l}\text { IG no nascimento, sema- } \\
\text { nas, média (DP) }\end{array}$ & 38,1 & $(0,9)$ & 38,2 & $(1,0)$ & $0,869^{*}$ \\
\hline $\begin{array}{l}\text { Peso do RN, gramas, } \\
\text { média (DP) }\end{array}$ & $3.913,0$ & 363,6 & $2.970,5$ & 391,9 & $<0,000^{*}$ \\
\hline \multicolumn{6}{|l|}{ Tipo de Parto, n (\%) } \\
\hline Parto vaginal & \multicolumn{2}{|c|}{$1(10,0 \%)$} & \multicolumn{2}{|c|}{$7(21,9 \%)$} & $0,655^{* *}$ \\
\hline Parto cesáreo & \multicolumn{2}{|c|}{$9(90,0 \%)$} & \multicolumn{2}{|c|}{$25(78,1 \%)$} & \\
\hline \multicolumn{6}{|l|}{ Sexo do RN, n (\%) } \\
\hline Feminino & \multicolumn{2}{|c|}{$3(30,0 \%)$} & \multicolumn{2}{|c|}{$17(53,1 \%)$} & $0,284^{* *}$ \\
\hline Masculino & \multicolumn{2}{|c|}{$7(70,0 \%)$} & \multicolumn{2}{|c|}{$15(46,9 \%)$} & \\
\hline \multicolumn{6}{|c|}{ Apgar de $1^{\circ}$ minuto, $n(\%)$} \\
\hline$\leq 7$ & 0 & $(0 \%)$ & 3 & $(9,4 \%)$ & $1,0^{* *}$ \\
\hline$>7$ & 10 & $(100 \%)$ & 29( & $(90,6 \%)$ & \\
\hline
\end{tabular}

${ }^{*}$ Teste de Mann-Whitney U. **Teste exato de Fisher. GIG = grande para a idade gestacional; IG = idade gestacional; DP = desvio padrão.

Os resultados da cardiotocografia computadorizada e a análise dos parâmetros de avaliação da FCF estão expostos na Tabela 2. Em todos os casos, a interpretação do traçado pelo sistema computadorizado atingiu os critérios de normalidade. Não foram observadas diferenças significativas quanto à freqüência de movimentos fetais ou da FCF basal entre os grupos. Quanto à presença de acelerações da FCF com amplitude de 10 ou de $15 \mathrm{bpm}$ não houve diferença entre os grupos analisados. Entretanto, a análise da freqüência de acelerações (>15 bpm a cada 10 minutos) demons- 
trou que no grupo de fetos que apresentaram diagnóstico de GIG, esse tipo de aceleração foi menos freqüente $(0,8$ acelerações $/ \mathrm{min})$ quando comparados aos não GIG (1,5 acelerações $/ \mathrm{min}, \mathrm{p}=0,04$, teste de Mann-Whitney U).

Tabela 2 - Parâmetros da cardiotocografia computadorizada realizada no termo em casos de diabete melito pré-gestacional de acordo com a adequação do peso do recémnascido.

\begin{tabular}{|c|c|c|c|}
\hline & \multicolumn{3}{|c|}{ Adequação do peso do RN } \\
\hline & $\begin{array}{c}\text { GIG } \\
(n=10)\end{array}$ & $\begin{array}{l}\text { Não GIG } \\
(n=32)\end{array}$ & $\mathrm{p}$ \\
\hline $\begin{array}{l}\text { Atinge critério de normalida- } \\
\text { de, } n(\%)\end{array}$ & $10(100 \%)$ & $32(100 \%)$ & - \\
\hline $\begin{array}{l}\text { Freqüência de MF, movi- } \\
\text { mentos/hora, média (DP) }\end{array}$ & $38,8 \quad(47,4)$ & $52,0 \quad(51,4)$ & $0,575^{*}$ \\
\hline $\begin{array}{l}\text { FCF basal, bpm, média } \\
\text { (DP) }\end{array}$ & $140,7 \quad(14,0)$ & $140,1 \quad(9,2)$ & $0,929^{*}$ \\
\hline $\begin{array}{l}\text { Presença de acelerações } \\
>10 \text { bpm, } n(\%)\end{array}$ & $10(100 \%)$ & $32(100 \%)$ & \\
\hline $\begin{array}{l}\text { Freqüência de acelerações } \\
\text { >10 bpm/10min, média (DP) }\end{array}$ & $1,6 \quad(1,0)$ & $2,4 \quad(1,6)$ & $0,131^{*}$ \\
\hline $\begin{array}{l}\text { Presença de acelera- } \\
\text { ções>15 bpm, n (\%) }\end{array}$ & $7(70 \%)$ & $29(90,6 \%)$ & $0,135^{\star \star}$ \\
\hline $\begin{array}{l}\text { Freqüência de acelerações } \\
>15 \text { bpm/10min, média (DP) }\end{array}$ & $0,8 \quad(0,9)$ & $1,5 \quad(1,3)$ & $0,04^{*}$ \\
\hline $\begin{array}{l}\text { Duração dos episódios de } \\
\text { alta variação em porcenta- } \\
\text { gem, média (DP) }\end{array}$ & $42,7 \quad(21,3)$ & $42,1 \quad(22,3)$ & $0,859^{*}$ \\
\hline $\begin{array}{l}\text { Variação da FCF nos episó- } \\
\text { dios de alta variação (bpm), } \\
\text { média (DP) }\end{array}$ & $16,2 \quad(2,5)$ & $19,7 \quad(4,2)$ & $0,02^{*}$ \\
\hline $\begin{array}{l}\text { Duração dos episódios de } \\
\text { baixa variação em porcen- } \\
\text { tagem, média (DP) }\end{array}$ & $13,2 \quad(19,1)$ & $20,9 \quad(26,2)$ & $0,407^{*}$ \\
\hline $\begin{array}{l}\text { Variação de curto prazo } \\
\text { (ms), média (DP) }\end{array}$ & $8,3 \quad(2,2)$ & $8,3 \quad(2,4)$ & $0,988^{*}$ \\
\hline
\end{tabular}

$\mathrm{GIG}=$ grande para a idade gestacional; $\mathrm{MF}=$ movimentos fetais; $F C F=$ freqüência cardíaca fetal; DP = desvio padrão. *Teste de Mann-Whitney U. ${ }^{* *}$ Teste exato de Fisher.

A duração dos episódios de alta variação e baixa variação pela cardiotocografia computadorizada não demonstrou diferenças, quando analisada em proporção ao tempo total do exame. Nos episódios de alta variação, a FCF demonstrou variação $(\mathrm{bpm})$ significativamente inferior nos fetos que apresentaram diagnóstico de GIG (variação de $16,2 \mathrm{bpm}$ ) quando comparados aos não GIG (variação de 19,7 bpm, p=0,02, teste de Mann-Whitney U). Quanto à variação de curto prazo, em ms, não houve diferenças significativas entre os dois grupos analisados. Estes dados estão demonstrados na Tabela 2.

\section{Discussão}

O controle clínico materno em gestações com diabete melito tem proporcionado redução na incidência de alterações nos testes de vitalidade que caracterizam o sofrimento fetal ${ }^{13}$. Entretanto, a elevada incidência de recém-nascidos GIG em gestações complicadas pelo diabete reflete a necessidade de maior conhecimento das alterações desencadeadas pela estimulação do crescimento excessivo no organismo fetal ${ }^{4}$.

A hiperinsulinemia tem sido considerada o problema básico do feto de mãe diabética ${ }^{1}$. Resulta do estado de hiperglicemia materna e estimula o crescimento fetal, tanto pela maior oferta de glicose para a circulação como pela regulação dos fatores de crescimento ${ }^{4}$. Essas substâncias atuam sobre outros fatores placentários, tais como os transportadores de aminoácidos, aumentando sua expressão e atividade, facilitando o transporte de nutrientes pela barreira placentária. Aumenta também a deposição de glicogênio e de lípides nos tecidos de armazenagem, como o figado, o músculo e o tecido adiposo ${ }^{14}$.

Além das alterações metabólicas e do crescimento fetal excessivo, são observadas anormalidades no desenvolvimento do coração dos fetos de mães diabéticas. Estudos demonstram espessamento do septo interventricular e das paredes dos ventrículos direito e esquerdo, mas não verificam alterações hemodinâmicas significativas ${ }^{15}$.

O potencial impacto do diabete materno no crescimento acelerado do coração fetal é pouco explorado nos estudos, principalmente do ponto de vista funcional deste órgão. Isso ocorre, muito provavelmente, pelas dificuldades em se realizar rotineiramente a avaliação das funções cardiacas fetais. Entretanto, a hipertrofia miocárdica pode justificar maior demanda energética do órgão, exigindo assim maior aporte local de oxigênio. Dessa forma, o órgão é mais suscetivel a hipoxemia, e as alterações funcionais precoces do comprometimento cardiaco podem ser melhor investigadas pela análise computadorizada da FCF.

No presente estudo, as características maternas e os resultados perinatais não apresentaram diferenças entre os grupos GIG e não GIG. Somente foram incluídos para análise os casos que atingiram o termo da gestação, pois a maturação do sistema nervoso parassimpático é influenciada pela idade gestacional, podendo interferir com a regulação da FCF. Para a comparação dos grupos deste estudo, foi importante assegurar que os fetos haviam sido analisados na mesma fase gestacional, no termo. Não foram verificadas diferenças quanto à idade gestacional no nascimento e quanto ao tipo de parto realizado, fatores esses que 
poderiam interferir na interpretação dos resultados. De forma semelhante, não se observou diferença quanto aos índices de Apgar dos recém-nascidos, que poderiam refletir maior comprometimento agudo da oxigenação fetal.

É relatado também que os fetos de mães diabéticas apresentam retardo na maturação de órgãos e sistemas ${ }^{16}$, que é mais evidente em gestações com descontrole metabólico importante. Dentro deste contexto, a cardiotocografia computadorizada passa a representar nova metodologia de pesquisa, permitindo a análise de parâmetros da FCF que refletem a interação dos sistemas simpático e parassimpático, que podem estar alterados pelo retardo na maturação do sistema nervoso autônomo fetal. O sistema 8002 permite análise detalhada da FCF, avaliando aspectos que não são contemplados na análise visual. Os parâmetros relacionados à variação da FCF são analisados com maior objetividade, permitindo a detecção de diferentes padrões que possam refletir a atividade neurológica fetal. Entretanto, neste estudo realizado em fetos a termo, não foram constatadas diferenças nos valores da FCF basal que pudessem refletir retardo na maturação do parassimpático, em que seria esperado observar freqüências mais elevadas. A pesquisa em idades gestacionais mais precoces talvez possa demonstrar, ao longo da gestação de fetos de mães diabéticas, a influência dos estímulos hiperglicemiantes no retardo da maturação autonômica fetal.

Poucos são os estudos acerca da cardiotocografia computadorizada em gestantes diabéticas. Serra-Serra et al. ${ }^{17}$ avaliaram os efeitos da hiperglicemia pós-prandial na FCF investigada pela cardiotocografia computadorizada em gestantes diabéticas e não verificaram alterações significativas nos resultados observados. No entanto, esses autores não analisaram os efeitos do descontrole metabólico sobre o exame fetal, e sim as eventuais alterações diante de um episódio pontual de hiperglicemia. Sabe-se que alterações da função cardiaca fetal podem surgir em fetos de mães diabéticas submetidos, em períodos precoces da vida intrauterina, a regime de hiperglicemia ${ }^{14}$.

As alterações observadas no presente estudo demonstram redução na variação da FCF analisada nos episódios de alta variação identificados pela cardiotocografia computadorizada, de forma semelhante ao que é observado na análise visual da cardiotocografia, em que a redução da variabilidade da FCF pode significar maior suscetibilidade à hipoxemia que esses fetos apresentam. Weiner et al. ${ }^{10}$ demonstraram resultados semelhantes, pois realizaram estudo da cardiotocografia computadorizada em 120 gestantes diabéticas, das quais 21 enquadravam-se na categoria pré-gestacional, e observaram redução na variação da FCF na $38^{a}$ se- mana de gestação nos fetos de mães diabéticas, quando comparados aos fetos de gestantes normais.

Há poucos estudos que analisam a FCF pela cardiotocografia computadorizada em gestantes com diabete, para que os resultados aqui apresentados possam ser comparados efetivamente. Estudos experimentais demonstram que a insulina exerce importante papel na regulação do desenvolvimento cardíaco fetal ${ }^{18}$. Isso se relaciona à hipertrofia cardiaca observada em filhos de mães diabéticas quando submetidos a regime de hiperinsulinemia durante a vida intra-uterina ${ }^{15}$, o que poderia levar a alterações na variabilidade da FCF.

Tincello et al..$^{8}$, em análise retrospectiva das cardiotocografias computadorizadas realizadas em 40 gestantes com diabetes melito insulino-dependente, destacam a ausência de episódios de alta variação nos fetos de mães diabéticas quando comparados aos fetos de gestantes normais. No entanto estes casos não são avaliados sob a ótica do descontrole glicêmico das gestantes e os autores também não fizeram comparações com a ocorrência de fetos GIG. Esse mesmo grupo ${ }^{9}$ realizou estudo prospectivo com 26 gestantes diabéticas tipo 1 , e relacionou as alterações observadas na cardiotocografia computadorizada com o retardo na maturação fetal, demonstrando novamente menor freqüência de episódios de alta variação em gestantes diabéticas quando comparadas ao grupo normal ${ }^{9}$. Contudo, nos resultados neonatais descritos, os autores compararam os dados do recémnascido de acordo com a presença ou não dos episódios de alta variação. Verifica-se no grupo com episódios de alta variação maior média do peso dos recém-nascidos, apesar de menor média na idade gestacional no nascimento, sugerindo maior proporção de recém-nascidos grandes.

No presente estudo foi observada menor freqüência de acelerações (15 bpm) da FCF nos fetos que se apresentaram GIG no nascimento. Resultado semelhante é relatado por Weiner et al. ${ }^{10}$, que verificaram menor freqüência de acelerações da FCF na $34^{\mathrm{a}}$ e na $38^{\mathrm{a}}$ semana dos fetos de mães diabéticas, quando comparados aos fetos de gestantes normais. A presença de acelerações é um dos marcadores cardiotocográficos que tradicionalmente caracterizam o bem-estar fetal. A redução pode associar-se a fetos com maior risco para a hipoxemia e acidemia no nascimento ${ }^{19}$.

O descontrole metabólico materno, resultando em maior exposição do feto a episódios de hiperglicemia e hiperinsulinemia, é responsável pelo aumento do metabolismo celular, o que provoca maior demanda de energia e incremento no consumo de oxigênio. A glicosilação da hemoglobina é responsável pelo deslocamento da curva de dissociação da oxi-hemoglobina para a esquerda, que resulta em maior afinidade da hemoglobina 
pela molécula do oxigênio e, portanto, reduz a liberação do mesmo nos tecidos. Nestas situações, o feto torna-se extremamente suscetivel a hipoxemia e é de se esperar que sejam verificadas alterações nos padrões da FCF, o que pode indicar precocemente a instalação do sofrimento fetal ${ }^{20,21}$.

Dentro deste contexto, a cardiotocografia computadorizada passa a representar instrumento metodológico interessante para a avaliação do feto em desenvolvimento, em face de distúrbios que possam alterar os seus padrões normais. Os efeitos da hiperinsulinemia fetal sobre a regulação da FCF não demonstraram ser muito evidentes, pois não foram observadas alterações importantes na FCF basal.

Trata-se aqui de estudo exploratório em que o conhecimento específico sobre a influência do diabete na regulação da FCF ainda não está estabelecido. O número restrito de casos arrolados no presente estudo não permite conclusões definitivas, sugerindo que anormalidades em parâmetros específicos da cardiotocografia computadorizada associam-se a casos de maior descontrole glicêmico.

A maior freqüência de acelerações da FCF e a maior variação da FCF em episódios de alta variação são características esperadas em situações de melhor vitalidade fetal e foram associados aos fetos que se apresentaram não GIG ao nascimento. Esses aspectos verificados pela análise da cardiotocografia computadorizada pode refletir padrões indicativos de melhor condição de oxigenação fetal naqueles menos comprometidos pelos efeitos do diabete na gravidez.

\section{Referências}

1. Harman CR, Menticoglou SM. Fetal surveillance in diabetic pregnancy. Curr Opin Obstet Gynecol. 1997;9(2):83-90.

2. Jovanovic L, Knopp RH, Kim H, Cefalu WT, Zhu XD, Lee YJ, et al. Elevated pregnancy losses at high and low extremes of maternal glucose in early normal and diabetic pregnancy: evidence for a protective adaptation in diabetes. Diabetes Care. 2005;28(5):1113-7.

3. Clausen TD, Mathiesen E, Ekbom P, Hellmuth E, Mandrup-Poulsen T, Damm P. Poor pregnancy outcome in women with type 2 diabetes. Diabetes Care. 2005;28(2):323-8.

4. Landon MB. Obstetric management of pregnancies complicated by diabetes mellitus. Clin Obstet Gynecol. 2001;43(1):65-74.

5. Pardey J, Molden M, Redman CW. A computer system for the numerical analysis of nonstress tests. Am J Obstet Gynecol. 2002;186(5):1095-103.

6. Nomura RMY, Francisco RPV, Steinman DS, Miyadahira S, Zugaib M. Análise computadorizada da cardiotocografia anteparto em gestações de alto risco. Rev Bras Ginecol Obstet 2002;24(1):29-36.

7. Walkinshaw SA. Pregnancy in women with preexisting diabetes: management issues. Semin Fetal Neonatal Med. 2005;10(4):307-15.

8. Tincello DG, el-Sapagh KM, Walkinshaw SA. Computerised analysis of fetal heart rate recordings in patients with diabetes mellitus: the DawesRedman criteria may not be valid indicators of fetal well-being. J Perinat Med. 1998;26(2):102-6.

9. Tincello D, White S, Walkinshaw S. Computerised analysis of fetal heart rate recordings in maternal type I diabetes mellitus. BJOG. 2001;108(8):853-7.

10. Weiner Z, Thaler I, Farmakides G, Barnhard Y, Maulik D, Divon MY. Fetal heart rate patterns in pregnancies complicated by maternal diabetes. Eur J Obstet Gynecol Reprod Biol. 1996;70(2):111-5.

11. Buinauskiene J, Baliutaviciene D, Zalinkevicius R. Glucose tolerance of 2- to 5-yr-old offspring of diabetic mothers. Pediatr Diabetes. 2004;5(3):143-6.

12. Ramos JLA. Avaliação do crescimento intra-uterino por medidas antropométricas do recém-nascido [tese]. São Paulo: Universidade de São Paulo; 1983.

13. Nomura RMY, Francisco RPV, Maganha CA, Miyadahira S, Banduki Neto JD, Zugaib M. Vitalidade fetal em gestações complicadas com diabete melito pré-gestacional: um estudo longitudinal. Rev Bras Ginecol Obstet. 2002;24(2):113-20.

14. Illsley NP. Placental glucose transport in diabetic pregnancy. Clin Obstet Gynecol. 2000;43(1)116-26.

15.Jaeggi ET, Fouron JC, Proulx F. Fetal cardiac performance in uncomplicated and well-controlled maternal type I diabetes. Ultrasound Obstet Gynecol. 2001;17(4):311-5.

16. Scholl TO, Sowers M, Chen X, Lenders C. Maternal glucose concentration influences fetal growth, gestation, and pregnancy complications. Am J Epidemiol. 2001;154(6):514-20.

17. Serra-Serra V, Camara R, Sarrion P, Jareno M, Cervera J, Bellver J, et al. Effects of prandial glycemic changes on objective fetal heart rate parameters. Acta Obstet Gynecol Scand. 2000;79(11):953-7.

18. Belke DD, Betuing S, Tuttle MJ, Graveleau C, Young $\mathrm{ME}$, Pham $\mathrm{M}$, et al. Insulin signaling coordinately regulates cardiac size, metabolism, and contractile protein isoform expression. J Clin Invest. 2002;109(5):629-39.

19. Bradley RJ, Brudenell JM, Nicolaides KH. Fetal acidosis and hyperlacticaemia diagnosed by cordocentesis in pregnancies complicated by maternal diabetes mellitus. Diabet Med. 1991;8(5):464-8.

20. Strachan BK, Sahota DS, van Wijngaarden WJ, James DK, Chang AM. Computerised analysis of the fetal heart rate and relation to acidaemia at delivery. BJOG. 2001;108(8):848-52.

21. Salvesen DR, Freeman J, Brudenell JM, Nicolaides KH. Prediction of fetal acidaemia in pregnancies complicated by maternal diabetes mellitus by biophysical profile scoring and fetal heart rate monitoring. $\mathrm{Br} \mathrm{J}$ Obstet Gynaecol. 1993;100(3):227-33. 